\title{
What Services, or Work Units, Do Nurses Experience in a More Traumatic Way When Entering the Professional World in Tenerife, Spain? A Phenomenological Study
}

\author{
Cristo Manuel Marrero González \\ University of La Laguna
}

\author{
Alfonso Miguel García Hernández \\ University of La Laguna
}

\begin{abstract}
The aim of this research is to investigate in which units or services the incorporation of nurses in Tenerife (Spain), is lived in a more difficult or traumatic way. In order to do so, a phenomenological hermeneutic investigation was carried out, by conducting an anonymous and confidential interview with nurses who have worked in public or private health institutions on the island of Tenerife and who have graduated from the University of La Laguna between 2009 and 2014. Three categories of the results of the interview data collection are established: ICU, OR and pediatrics. The incorporation to these services is often done in a hasty manner, without previous preparation of the nurses in these services. The participants in this study state they need support and better integration in these complex units.
\end{abstract}

Keywords: novice nurse, job incorporation, stress, phenomenology

\section{INTRODUCTION}

Patricia Benner describes the different stages a nurse goes through at the professional level according to her model of professionalization and learning, which has become a reference in different studies. The author refers that a novice nurse is "an inexperienced nurse with limited, inflexible performance, governed by out-of-context rules and regulations rather than by experience". Although this definition usually refers to the student nurse, the lack of experience of a newly graduated nurse in an unfamiliar work context puts her back in a student situation. Many novice nurses have to experience new situations that they have never experienced before, and they have to cope with them. It takes two to three years for nurses to become competent and confident in their practice. Novice nurses act on abstract principles, conventional models, and theories to engage in a new situation so that they can learn safely and effectively (Benner, 1987).

Unlike veteran nurses, novices have organized knowledge as separate facts. They rely on resources such as books, notes or tutors, but do not have the knowledge acquired through experience. In fact, novices present obstacles due to lack of knowledge of resources and loss of mental capacity due to anxiety, not being in control of a particular situation, and lack of self-confidence. New practitioners feel uncomfortable if the patient's needs do not involve performing a procedure exactly as they learned it. 
Context or circumstances are an important part of critical thinking in nursing because such thinking changes according to circumstances. Not knowing a certain context, or not having enough experience in that context, causes critical thinking in the nurse to become impaired. An example is outlined above when a nurse who is an expert in one area works in a specialty that is unfamiliar to the nurse. Therefore, it is important to pay attention to the context in which the nurse is working. (Benner, 1987; Alfaro-LeFevre, 2009: 69-72).

In addition, there is a "culture shock" to assimilate the challenges and the "new culture", or situation that they have to live, and the identification of barriers to successfully carry out the transition process from student to professional, in addition to the endoculturation that this fact entails. Some of these barriers are related to the work environment, frustration and stress, inadequate preparation or lack of social support. (Meyer and $\mathrm{Xu}, 2005$; Valdez, 2008).

Having stated these premises about the professional limitation of the new or recently graduated nurse, and the fact that a recently graduated nurse in Spain can work in Spain in different work units without taking into account their preparation or experience (Blanco García, 2012).

\section{OBJECTIVES}

- To find out which are the work units that nurses graduated from the University of La Laguna (Tenerife, Spain) between 2009 and 2014 experience in a more traumatic way when they start working in different health institutions in Tenerife, both public and private.

- To describe and analyze the experiences of the nurses within the units that they consider the hardest when starting to work in the health context of Tenerife.

\section{METHODS AND MATERIALS}

A qualitative research is carried out, with a hermeneutic phenomenological approach: narrative study (discourse analysis). The nurses participating in this research are graduates of the University of La Laguna between 2009 and 2014 and have worked in health institutions on the island of Tenerife, both private and public. Thirteen professionals graduated from said university between those years participate: 11 women and 2 men. The selection of this sample was made randomly, as the authors contacted the participants to propose them to participate in the research, explaining the objectives of the study.

Data collection is conducted through the following semi-structured and brief interview "In which units or services did you feel most insecure? Why?" and "Were you afraid when you started working in a particular service?" The interviews are conducted personally and privately outside the healthcare institutions, so we only asked for consent from each participating nurse to be interviewed. Interviews are collected on audio recorder and transcribed to Word format.

The narratives are treated through the selection, segmentation and coding of the data using the NVIVO 10 software. Thus, with the discourse analysis of the narratives obtained, the different categories of results are established. With the sample of participants with which the study has been carried out, a saturation of the data is obtained, making this qualitative research valid.

The work is carried out in the second half of 2015, being part of a more complex study in which the experiences of nurses who graduated from the University of La Laguna during their incorporation into the labor market are addressed.

\section{RESULTS}

The three categories that have been established after the collection of data from the interview with the participating nurses are developed below. 


\section{ICU}

For the new nurse, this unit is always a challenge not only because of the type of patients to be treated, but also because of the complexity of the specialty of intensive care: experience and skills are needed, it is necessary to know how to handle complex technology, it is necessary to know and be confident with the drugs to be administered and to know how to overcome the pressure of care and the work environment in complex situations.

Interpersonal relationships between co-workers in this unit or specialty are important because the nurse needs the support of the rest of her colleagues with more experience and training in the unit: the nurse has to learn quickly and safely because she is not sufficiently prepared to work in an intensive care unit.

Support from the rest of the colleagues is important for learning and for working safely. The new professional is hired as just another professional without taking into account her preparation, so she must constantly ask for help and support from the rest of her colleagues, which she does not always receive, due to the overload of the colleague, the stress experienced by the colleagues or simply, as the nurses say, because they do not want to help them or because they have negative attitudes towards them.

The environment described in a critical care unit is multifactorial: difficult, complex and strange. The demands placed on the professionals, together with the work overload, stress, etc., make the newly arrived nurse in this unit undergo an experience that is difficult to grasp when entering the context of an ICU.

"When you were sent to the ICU, they would say, 'It's okay, it's okay.' Then you would get there and say, 'How has the shift been?' 'Four patients have had a cardiac arrest' 'What? But I don't even know where the crash cart is, or where the defibrillator is.' I would have been grateful and paid for training, especially on the first day I started working in the ICU, which I started at night, and of course I had a hard time. I had no idea. I didn't know anything my last training. I had done it in a health center, and it is the freshest thing you usually remember, and I remember the guy told me: 'you have two options, you can either go downstairs and smoke a cigarette or have a cortado or go to the bathroom and cry. I recommend the first one, you take a walk and come back.' I was scared. I was crying. I didn't know how to do things, I didn't know how to set pumps. The pumps were beeping all the time. It drove my head crazy. I remember I finished at 8 o'clock and I went to the gastroenterology floor at 8 o'clock, where a cousin of mine works, and I spent the whole morning with him so he could explain to me how to set pumps. I would have paid to have three weeks or a month's training and have someone explain to me how the plant works. Even without getting paid." (Nurse 1)

"I don't see the ICU as closing its doors to me. In all the departments I have been in, you will always find all kinds of people who are willing to help, colleagues who are not so nice, colleagues who are willing to give you a hand, others who say: 'I am not here today to explain anything to you'. But in general in all places, and even more so in a hospital where there are so many people, there are always better or worse colleagues. But well, you have to try to adapt." (Nurse 2)

"If I were sent to the ICU, for example, I think I would be freaking out that day. Because after such a long time without being in contact with a patient that critical... It's like I say, they pick you up and they drop you there and that's it... I mean, you've never been there before." (Nurse 3)

"The ICU frightens me to death." (Nurse 4)

"I was not very satisfied. It was a difficult environment. The work environment in the ICU is a little tense at times. There are a lot of things to learn, a lot of information. I don't think some people are smarter than me, or that I'm dumber, if you've just arrived in the ICU and 
you have to do certain things, unless you're reckless, you don't feel comfortable doing some of the things I had to do. Even if they explain it to you it's a weird feeling." (Nurse 5)

"The most insecure service I think was the ICU. I don't think it's logical for me to have to manage a brain trauma patient even if I know how to calculate a Glasgow scale, but I think that in order to provide the quality of care, perhaps additional training and experience is needed." (Nurse 5)

"Of course, it is true that in special services there is always a little more stress because they provide more specialized treatment. I had to go to the ICU during my first hospital contracts. I was very nervous, but they welcomed me well. In fact, they gave me one of the best patients within their circumstances. But you realize that even if you have a patient who is relatively stable and so on, you realize what it's like to be in a special unit. You realize that you have to have a lot of training. People who have training find themselves in situations that overwhelm them as well." (Nurse 6)

"ICU. It causes me a lot of stress that the most critical patients can end up in the unit at any time or not knowing protocols or specific techniques. The problem lies in the fact that such a case may occur in the evening, where I am with the doctor on duty, and in case there is a problem on another floor, I must be the most 'experienced' one to go and solve it." (Nurse 7)

"The issue of mechanical ventilation, medication in critical care such as sedation, amines and all that I had not been trained in. The day I was called from being in primary care to being directly in the ICU: 'Come on, there you have two ICU patients. Two critical intubated patients for you'... That was terrible for me. I lived through that with a lot of anxiety, in fact, during those two months in the ICU I lost weight, I hardly ate. I would get home and hear the "beep beep" of the respirator. I had it so deep in my head that I could hear it when I slept. Luckily, I was with colleagues who more or less looked after me, but I was very scared and I also felt like a burden to them because of course you have to ask them everything. They are patients that slip away from you, it was a time I lived with fear, with anxiety, I had a bad time, but thanks to that I have also become a nurse and I have been trained, not in the way I would have liked, but by 'putting myself together', taking courses, asking questions, being ignorant because sometimes some colleagues do not support you because they say 'you get paid the same as me and I will not help you'." (Nurse 2)

"I know you feel alone and displaced in that environment. It is a unit that handles very critical patients. They are people who have been working for a long time, they have established their own teams and of course there are people who maybe have been working for 20 years and they see someone new and they are tired and they say 'I am not going to teach you, you are not a student. Fuck with the new one, now I have to handle not two but four patients because I have to keep an eye on her'." (Nurse 2)

"I think I had an anxiety crisis twice. I was 'this close' to saying I give up, I quit. You have two options: either you give up and sink, or you bounce back. Thank God I recovered. A horrible anxiety. One of the times because I felt abandoned by all my colleagues. I was new there and I had to do my job and even though I had no idea how it was done because I remember it was the ICU and aha! If you make a mistake you kill the patient. They had a very negative attitude towards me, 'Why do I have to teach you, you are already a nurse. 
It's not my fault and you will figure out what to do because if you mess up these patients are very delicate.' That was very bad." (Nurse 8)

\section{Operating Room}

Nurses do not feel prepared to work in an operating room unit. They feel that they have not been trained or have not received sufficient training in the operating room. When they are hired for this unit, the hiring is often done very quickly without taking into account the situation of the novice nurse. It is important that the nurse who starts working in the operating room has good colleagues to help the nurse.

"I did not feel prepared, no matter how hard I studied. I remember the first day I worked in the operating room reception unit. I had never been in the operating room reception, what the hell was I supposed to do there? I had no one to help me. You don't feel prepared. For the more normal units you might be, but for the more special units, you don't." (Nurse 8)

"In fact, where I have felt most insecure was in the operating room, but because I think that there is a huge gap between what you learn in your career and what it is like to work in an operating room. Because in the career they really do not teach you anything about the operating room. They talk to you about asepsis, they talk to you about some surgical concepts, but little else. And then you are called to work in the operating room, not as a support but as an instrumentalist, as happened to me. I had been working for about a year and I was called to the operating room for a week as an instrumentalist. The experience was terrible, because in addition to the typical thing that they call you at 14:30 to start at 15:00, it was a unit that you don't know at all." (Nurse 9)

"Well, in the operating room, because of the little (or no) experience I had in the service. I didn't really know what my role was there and the first day was quite hard, I felt that I couldn't contribute much." (Nurse 10)

"Yes, because my first contract, as I told you before, was in the operating room and they put me there, although I had been in the operating room and I had chosen the operating room tutoring in my career, it is not the same when you are alone, because when I was in my career I was in gynecology and this time I had an ENT, but well, they put a colleague there with me. It is true that during the two days I was in the operating room because later they changed me, they assigned me a partner to be with me and explain to me more or less." (Nurse 11)

\section{Pediatrics}

The nurses perceive insecurity and lack of preparation when working in the specialty of pediatrics at both hospital and primary care levels. It is a challenge for the participants because of how complex it is for them to treat a child since it is an unknown field and it generates insecurity due to the little training they have received. The nurses feel unprepared to work in a pediatric or neonatal intensive care unit, as well as to work in a primary care nursing practice: they report feeling panic for not having enough experience, for not knowing how to act in certain situations in pediatrics and for fear of making mistakes.

"Since I started in a place that was relatively easy because I did not have much technique or responsibilities, I was more or less managing, but it is true that when I moved to the hospital, and I started in pediatrics, it was a constant fear because many times I was paralyzed. I knew I had to do something, I knew how to do it, but I kind of avoided it. I avoided doing that cure, or going to make that change or going to do that because I panicked actually. If you already feel useless when you start, especially with a specialty like children, 
which is a world apart, and of course you have no training whatsoever, then you are already in big trouble because it has nothing to do with what you have studied, or what you are used to." (Nurse 9)

"Perhaps those that have to do with pediatrics do create a lot of stress for me, the issue of working with children because not only do you work with them but also with their parents and of course pediatrics are situations that depend on where you are, if you are in a pediatric ICU there are situations with children who are quite ill and have a very specialized treatment, the truth is that it would scare me very much if they told you that I had a contract in pediatrics in the pediatric ICU of the hospital or wherever because I did not know how to really act in this situation that of course creates stress for the parents and yourself as a professional and if you do not have experience it is a unit in which I feel quite insecure, especially since my time in pediatrics was very short during my training." (Nurse 10)

"Pediatrics. These are services that you have a lot of respect for precisely because of that. Perhaps due to lack of knowledge and because they are children, whether you like it or not. After all, that's what pediatrics is all about. Pediatrics is a service that scares me. You should not be forced to take it in many cases without training. It is a service that even in primary pediatrics I panic about. Don't ask why. I don't know if it's because of the bad experiences I had during my training, but there I got a lot of insecurity and a lot of panic." (Nurse 12)

"In primary care: in pediatrics, in my case, I did my training related to pediatrics in the neonatology unit in the second year of my career, so it was difficult to feel confident in the pediatrics office." (Nurse 13).

\section{DISCUSSION AND CONCLUSIONS}

One of the specialties in which nurses rotate a lot in Tenerife is undoubtedly the ICU. This unit is perceived by the nurse as a complex unit and difficult to adapt to due to the type of patient, the hasty incorporation to work in critical care, the lack of preparation, the sometimes-complicated work environment and the "stress" that it causes for the new nurse.

The nurses who work for the first time in a critical care unit value the accessibility and support of the rest of their colleagues due to positive reinforcement. In our study, interpersonal relationships within a critical care unit, and the environment of the same, are valued in an important way by the participating nurses because, as we have seen, they experience difficult situations due to the lack of support and this makes them not feel sufficiently prepared to work in a critical care unit.

Saghafi $(2012 ; 2014)$, refers that at the beginning the nurse feels oversaturated or included or excluded from the team depending on whether she has positive feedback or not with the team to work with. We agree with Dracup and Bryan-Brown (2004) and Farnell and Dawson (2006), in the importance given by new nurses to have adequate support in a critical care unit as they observe complexity at the level of socialization as shown in the results of the present work.

We agree with Gohery and Meaney (2013), in the fact that nurses are employed in critical care units without sufficient preparation and that this implies fear due to the responsibility and lack of sufficient training when entering a complex and stressful environment, so they need more support.

The incorporation to critical care units is a complex and hard event for the new nurse that requires the necessary social support within the unit so that the new nurse feels confident in her work. Joining critical care units should not be rushed, but gradual.

But it is not only in critical care units where we perceive that adapting to hospital work is a difficult experience. It is not only the pressure of care and work overload, but also human relations and work environments that play a role. In any hospitalization unit, novice nurses place great value on interpersonal 
relationships, especially on receiving support from the rest of the team. From what we can appreciate from the results of our interviews, entering the work context involves an experience clearly marked by the work environments. However, we observed that this is not usually the case in primary care, where there is a better integration in the work environment.

The operating room service is another of the special services that is significantly experienced in some nurses interviewed under circumstances very similar to those that occur when a nurse is hired for a critical care unit: lack of preparation, haste at the time of being hired for the operating room without knowing the service and the substantial importance of having support from the rest of the team to be able to work confidently. The novice nurse is not sufficiently prepared to work in a service such as the operating room and adequate preparation is needed before starting to work in this service (Mars, 2015).

Another specialty highlighted by the nurses in this study is pediatrics. The participants express "respect", "fear", insecurity and lack of preparation during their studies, in addition to the fact that practical training is not sufficient, not only to treat pediatric patients, but also because pediatrics is perceived as a complex specialty, to which is added the difficulty of dealing with parents as well. Hospital pediatrics is undoubtedly a complex specialty for the new nurse, which also requires the necessary support and acceptance by the rest of the colleagues. As far as primary care pediatrics is concerned, the manifestations are still related to the lack of practical preparation, the haste on the part of the institutions when it comes to hiring and the acceptance on the part of the team. The novice nurse is often fearful of facing unfamiliar situations (Delaney, 2003; Casey et al 2004; Valdez, 2008). The capabilities of novice nurses are limited by inexperience, anxiety, and limitation in developing clinical judgments (Benner, 1987; Ferguson and Day, 2007; Alfaro-LeFevre, 2009).

\section{ACKNOWLEDGEMENT}

Translated \& edited by American Publishing Services (https://americanpublishingservices.com/).

\section{REFERENCES}

Alfaro-LeFevre, R. (2009). Pensamiento crítico y juicio clínico en enfermería: Un enfoque práctico para un pensamiento centrado en los resultados (Critical thinking and clinical judgment in nursing: A practical approach to outcome-focused thinking) (4th Ed). Elsevier Masson.

Benner, P. (1987). Práctica progresiva en enfermería: Manual de comportamiento profesional (Progressive nursing practice: A handbook of professional behavior) (1st Ed.). Barcelona: Enfermería y sociedad. Ediciones Grijalbo, SA.

Blanco García, B. (2012). Los Niveles de Estrés de los Estudiantes de Enfermería: Programas de Ayuda y Acogida para los Nuevos Profesionales de Enfermería (Trabajo fin de Máster) (The Stress Levels of Nursing Students: Support and Welcoming Programs for New Nursing Professionals (Master's thesis)). University of Oviedo (Spain).

Casey, K., Fink, R.R., Krugman, A.M., \& Propst, F.J. (2004). The graduate nurse experience. Journal of Nursing Administration, 34(6), 303-311.

Delaney, C. (2003). Walking a fine line: Graduate nurses' transition experiences during orientation. The Journal of Nursing Education, 42(10), 437.

Dracup, K., \& Bryan-Brown, C.W. (2004). From novice to expert to mentor: Shaping the future. American Journal of Critical Care, 13(6), 448-450.

Farnell, S., \& Dawson, D. (2006). 'It's not like the wards'. Experiences of nurses new to critical care: A qualitative study. International Journal of Nursing Studies, 43(3), 319-331.

Ferguson, L.M., \& Day, R.A. (2007). Challenges for new nurses in evidence-based practice. Journal of Nursing Management, 15(1), 107-113.

Gohery, P., \& Meaney, T. (2013). Nurses' role transition from the clinical ward environment to the critical care environment. Intensive and Critical Care Nursing, 29(6), 321-328. 
Marsh, V. (2015). Mentoring the novice OR nurse. AORN Journal, 102(2), 12-13. doi:10.1016/S00012092(15)00619-5

Meyer, T., \& Xu, Y. (2005). Academic and clinical dissonance in nursing education: Are we guilty of failure to rescue? Nurse Educator, 30(2), 76-79.

Saghafi, F., Hardy, J., \& Hillege, S. (2012). New graduate nurses' experiences of interactions in the critical care unit. Contemporary Nurse, 42(1), 20-27.

Saghafi, F. (2014). The journey from new graduate to proficient nurse in the Intensive Care Unit: A descriptive phenomenological study (Doctoral dissertation). Australian Catholic University.

Valdez, A.M. (2008). Transitioning from novice to competent: what can we learn from the literature about graduate nurses in the emergency setting? Journal of Emergency Nursing, 34(5), 435-440. 